\section{An unusual cause of dysphagia}

\section{Vishal Sharma, Surinder S. Rana}

Post Graduate Institute of Medical Education and Research (PGIMER), Chandigarh, India

A 42-year-old male presented with 2 months history of progressive dysphagia predominantly to solids. Computed tomography of the chest showed a large subcarinal lymph node (Fig. 1A). His PPD skin test was positive ( $32 \mathrm{~mm} \times 30$ $\mathrm{mm}$ ). He underwent gastroduodenoscopy where a submucosal bulge with normal overlying mucosa causing luminal narrowing was noted in mid-esophagus. Endoscopic ultrasound (EUS) revealed a large $(6 \mathrm{~cm} \times 4.5 \mathrm{~cm})$ subcarinal lymph node with few hyperechoic areas (Fig. 1B). Multiple lymph nodes were also seen in the upper mediastinum. EUS-guided fineneedle aspiration was done from the subcarinal lymph node that yielded caseous material. The cytological examination of the aspirate showed granulomatous inflammation and the stain for acid fast bacilli was negative. He was started on four drug antitubercular therapy and responded with resolution of dysphagia and weight gain.

Dysphagia is an uncommon presentation of tuberculosis [1]. Esophageal tuberculosis may mimic esophageal malignancy. It is most commonly recognized as an extrinsic bulge on endos-
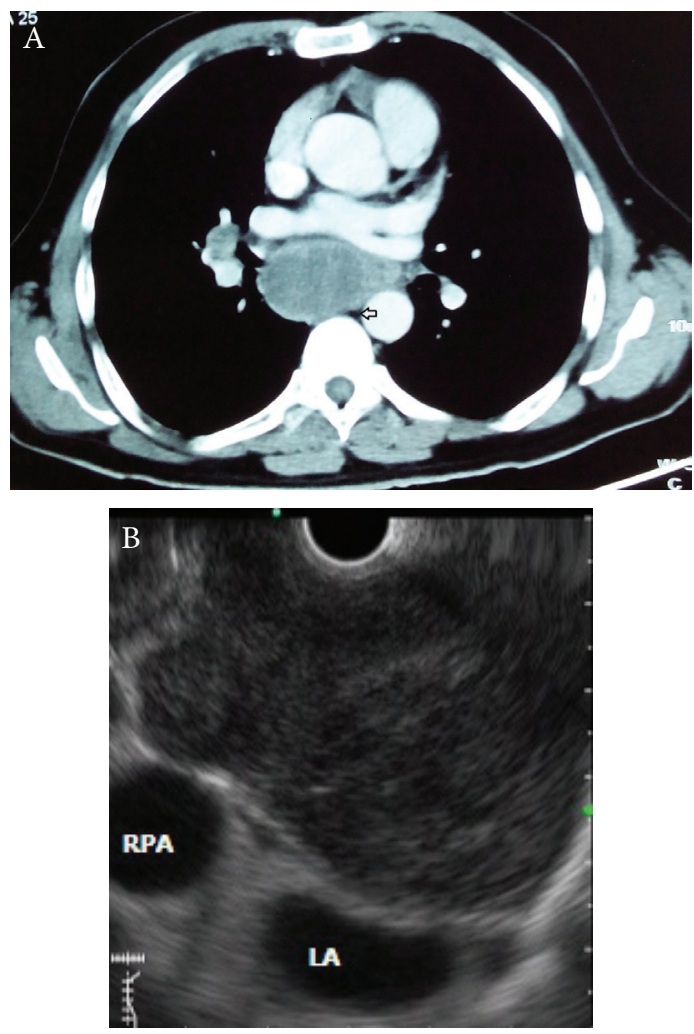

Figure 1 (A) Computed tomography showing enlarged subcarinal lymph node compressing the esophagus (arrow) (B) Endoscopic ultrasound: Large subcarinal lymph node compressing the left atrium $L A$, left atrium; RPA, right pulmonary artery copy. Other endoscopic features include ulcers and polypoidal lesions. The most common site of esophageal tuberculosis is mid-esophagus [2]. EUS provides the opportunity to detect and sample these lesions and has emerged as tool of choice to diagnose esophageal tuberculosis. The presence of hyperechoic foci, patchy anechoic or hypoechoic areas in the lymph nodes on EUS suggest tubercular etiology [3]. The response to four-drug combination therapy is rapid and the resolution of dysphagia is usually noted within 3 weeks of initiation of therapy [2].

\section{References}

1. Rana SS, Bhasin DK, Sharma V, Chaudhary V, Singh K. Dysphagia as the firstmanifestation of tuberculosis. Endoscopy 2011;43(Suppl 2) UCTN:E300-E301.

2. Rana SS, Bhasin DK, Rao C, Srinivasan R, Singh K. Tuberculosis presenting as dysphagia: Clinical, endoscopic, radiological and endosonographic features. Endosc Ultras 2013;2:92-95.

3. Rana SS, Bhasin DK, Srinivasan R, Singh K. Endoscopic ultrasound (EUS) features of mediastinal tubercular lymphadenopathy. Hepatogastroenterology 201;58:819-823.

Department of Gastroenterology, Post Graduate Institute of Medical Education and Research (PGIMER), Chandigarh, India

Conflict of Interest: None

Correspondence to: Dr Surinder Singh Rana, Ass. Professor, Department of Gastroenterology, Postgraduate Institute of Medical Education and Research (PGIMER), Chandigarh, 160012 India, Tel.: +91 172 2749123, Fax: +91 172 2744401,

e-mail: drsurinderrana@yahoo.co.in, sonalisurinder@yahoo.co.in

Received 24 September 2013; accepted 30 September 2013 